\title{
Evaluation of clinical datasets in fully automatic planning assist system for cardiac magnetic resonance imaging
}

\author{
Shuhei Nitta ${ }^{1 *}$, Taichiro Shiodera ${ }^{1}$, Tomoyuki Takeguchi ${ }^{1}$, Shigehide Kuhara² ${ }^{2}$ Kenichi Yokoyama ${ }^{3}$, Rieko Ishimura ${ }^{3}$, \\ Toshiaki Nitatori ${ }^{3}$
}

From 16th Annual SCMR Scientific Sessions

San Francisco, CA, USA. 31 January - 3 February 2013

\section{Background}

Planning assist systems for cardiac MR examinations are necessary for easier operation and shorter examination times. Slice alignment systems [1] have played an important role in achieving these objectives. We propose a new automatic planning assist system for couch adjustment, local shimming, and axial multislice imaging as the input to a slice alignment system [2]. The new system employs an atlas-based segmentation technique using single scout volume data. In the present study, the accuracy and robustness of the proposed method were evaluated based on more than 50 datasets including clinical data, and the results were compared against the degree of interobserver error in manual annotation.

\section{Methods}

An ECG-non-gated 3D fast field echo (FFE) single volume covering the entire chest area was acquired using a 1.5T MRI scanner (Excelart VantageTM powered by Atlas, Toshiba Medical Systems) during a single breathhold with TR/TE $=3.7 / 1.3$, FOV $=500 \times 350 \times 350 \mathrm{~mm}^{3}$ (coronal slab), and readout/phase/slice encode steps = 256/64/35 in an acquisition time of approximately 9 seconds. Our proposed method is based on a registration technique composed of several steps to achieve good accuracy with acceptable processing cost. First, the input volume is normalized in contrast by a histogram expansion. Then, the volume is transformed to match a prepared model volume with manual annotation of the heart region, permitting the heart region of the input data to be located. Finally, the result is modified by several image

${ }^{1}$ Corporate Research \& Development Center, Toshiba Corporation, Kawasaki, Japan

Full list of author information is available at the end of the article processing techniques. Accuracy was assessed by measuring the Euclidean distances of the six sides of the circumscribed cuboids of the cardiac area obtained by our method and by manual annotation, and evaluation was performed by comparison with the differences between two manual annotations as a measure of interobserver error.

\section{Results}

The proposed method successfully segmented the entire heart region for 48 datasets from 15 healthy volunteers and 51 datasets from 32 patients. The processing time was approximately 1.6 seconds $(2.5 \mathrm{GHz} \mathrm{CPU}$, singlethread processing). The average distance error for the left, right, anterior, posterior, head, and foot sides were $3.91 \pm 3.28,3.10 \pm 2.43,4.31 \pm 3.32,6.80 \pm 5.76,10.42 \pm 8.96$, and $6.15 \pm 5.12 \mathrm{~mm}$, respectively. The interobserver errors for 15 datasets from 15 healthy volunteers and 32 datasets from 32 patients were $3.61 \pm 2.68,3.25 \pm 3.09$, $4.31 \pm 4.39,5.77 \pm 3.97,13.22 \pm 8.52$, and $4.42 \pm 3.18 \mathrm{~mm}$, respectively.

\section{Conclusions}

We propose a new cardiac alignment system. The results showed that, even in patient data, the entire heart region could be detected by our method almost as accurately as by manual annotation. The proposed method combined with our previous work [2] should prove to be clinically useful as a fully automatic planning assist system for cardiac MRI.

\section{Funding}

No funding was received for this research. 

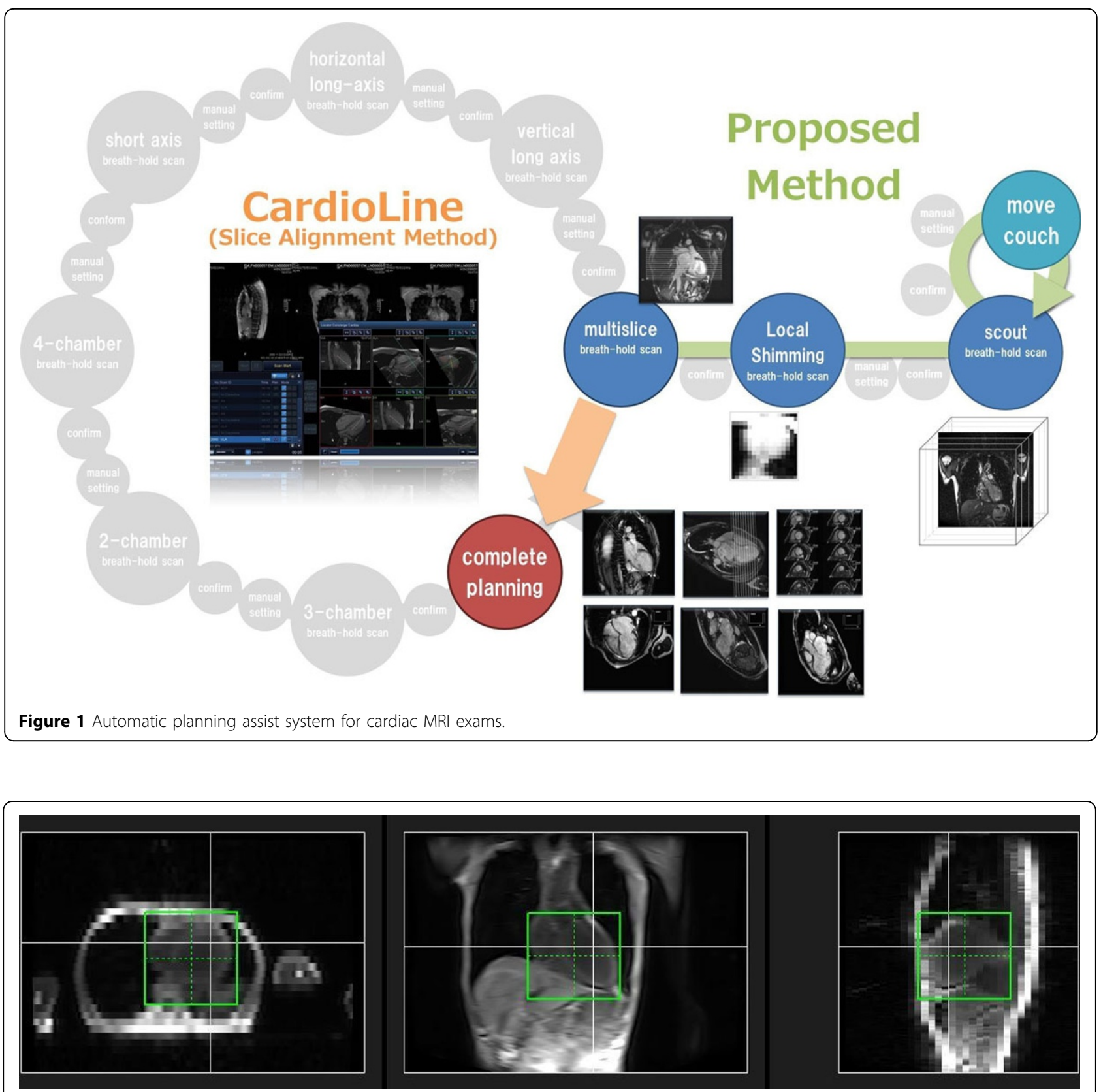

Figure 2 Example of detection results for actual clinical data.

\section{Author details}

'Corporate Research \& Development Center, Toshiba Corporation, Kawasaki, Japan. ${ }^{2}$ MRI Systems Division, Toshiba Medical Systems Corporation, Otawara, Japan. ${ }^{3}$ Department of Radiology, Kyorin University, Faculty of Medicine, Mitaka, Japan.

Published: 30 January 2013

\section{References}

1. Nitta, et al:. SCMR 2012, Poster ID: P269.

2. Nitta, et al:. ESMRMB 2012, No. 761.
doi:10.1186/1532-429X-15-S1-P33

Cite this article as: Nitta et al.: Evaluation of clinical datasets in fully automatic planning assist system for cardiac magnetic resonance imaging. Journal of Cardiovascular Magnetic Resonance 2013 15(Suppl 1): P33. 